\title{
FIFTY YEARS OF QUANTUM CHEMISTRY
}

\author{
E. BRIGHT WILSON \\ Department of Chemistry, Harvard University, 12 Oxford St., Cambridge, MA 02138, USA
}

\begin{abstract}
An assessment of some aspects of the impact of quantum mechanics on chemistry will be attempted, with emphasis on the different ways it has had an important influence. These include a change in the chemists' vocabulary, his way of looking at molecules and reactions, the instruments and methods he uses, the psychological satisfaction he gets from his subject, the procedures he uses to describe and to rationalize his results, as well as direct, partly predictive theories and numerical calculations. The introduction and improvement of the electronic computer played a major role but also diverted talent away from the development of new approaches. A number of other questions about quantum chemistry will be raised and personal answers offered.
\end{abstract}

Fifty years should be long enough for a new approach to have a major impact on a science, and there is no doubt about the magnitude of the impact of quantum chemistry. Quantum mechanics as applied to chemistry has an astonishing history. Only three years after its first formulation in 1926 Dirac made his famous statement ${ }^{1}$ "The underlying physical laws necessary for the mathematical theory of a large part of physics and the whole of chemistry are thus completely known ...", yet at that time not a single accurate chemical i.e. molecularapplication of quantum mechanics had been made. Nevertheless, Dirac's views have been shared ever since by most theoretical chemists, despite the fact that even today with mammoth computers not even dreamed of in 1929 , only a few properties of a handful of the most trivial molecules can be calculated with an accuracy approaching experiment. How can one say that scientists lack faith!

I wish now to discuss the impact of quantum mechanics on chemistry by asking - and then attempting to answera series of questions. Some of the answers will be personal judgments with which not everyone will agree.

The first question is: Is quantum mechanics correct? The answer is: surely not. Relativity is not properly taken into account, and of course the physicist has gone beyond ordinary elementary quantum mechanics in order to deal with high energy phenomena, appearing and disappearing particles, quantized radiation fields, etc.

Is ordinary quantum mechanics good enough for chemistry? For the lighter elements and most phenomena of chemistry, I would vote "yes". For the heavier elements, there still seem to be unresolved problems with relativistic effects. Table 1 shows some estimated corrections. ${ }^{2}$ These suggest that not all is well with heavier elements, although recent non-relativistic calculations on lead oxide ${ }^{3}$ have been interpreted as supporting the hypothesis that such calculations can be used.

Why should we believe that quantum mechanics is in

Table 1. Relativistic corrections for the ground state of some atoms. ${ }^{2}$ (Hartrees, 1 Hartree $=627.5 \mathrm{kcal}$ )

\begin{tabular}{lccc}
\hline Atom & Total energy & $\begin{array}{c}\text { Relativistic } \\
\text { correction }\end{array}$ & $\begin{array}{c}\text { Correlation } \\
\text { energy }\end{array}$ \\
\hline $\mathrm{He}$ & -2.90373 & -0.00007 & -0.0420 \\
$\mathrm{C}$ & -37.8561 & -0.01381 & -0.1551 \\
$\mathrm{Ne}$ & -129.051 & -0.13121 & -0.381 \\
$\mathrm{Si}$ & -289.906 & -0.58351 & -0.494 \\
$\mathrm{Ar}$ & -529.255 & -1.76094 & -0.732 \\
\hline
\end{tabular}

principle accurate, even for the lighter elements? Ideally, I should be able to quote a long list of molecules for which accurate energies, dissociation energies, ionization potentials, electron affinities, structures, dipole moments, etc. have been calculated and found to be in agreement with experiment to within the experimental error. For energies, I cannot do this. Despite the enormous computational power which is available, the work of James and Coolidge $\mathrm{e}^{4}$ on the hydrogen molecule with desk computers in 1936 is still the landmark. This is not to disparage the magnificent calculations of Kolos and Wolniewitz, who have indeed carried $\mathrm{H}_{2}$ to the very limits of modern experimental accuracy. ${ }^{6}$ See Table 2.

On the other hand the fact that completely nonempirical a priori treatments, necessarily involving serious approximations, come as close to experiment as many do is a considerable triumph and surely strong support for our belief in the ultimate correctness of quantum mechanics for the chemistry of light elements.

There is, moreover, another and perhaps more impressive test. The rotational energy levels of large numbers of polyatomic molecules can be determined from microwave spectroscopy to one part in a million. The lower levels can be fitted to the quantum mechanical formulas for the rigid rotor model by adjusting three parameters, the three principal moments of inertia. After three levels are used to set the parameters, there are many left over as a test of the fit, and the agreement is typically within a part in 30,000 or better for a large number of molecules. Further, no problems have arisen in understanding and fitting transition intensities, effects of electrical and magnetic fields, isotope shifts, nuclear spin weights, centrifugal distortion, quadrupole coupling, hyperfine structure, various tunnelling effects, and many others. Quantum mechanics seems completely adequate to fit these phenomena and then to make verifiable and successful predictions.

Can quantum mechanical calculations replace experi-

Table 2. Calculated and observed values for the dissociation energy $\left(\mathrm{D}_{0}{ }^{\circ}\right)$ of $\mathrm{H}_{2}$ (in $\mathrm{cm}^{-1}, 1 \mathrm{~cm}^{-1}=2 \cdot 8591 \mathrm{cal} / \mathrm{mol}$ )

\begin{tabular}{lcc}
\hline & Obs. $^{6}$ & Calc. $^{5}$ \\
\hline $\mathrm{H}_{2}$ & $36117 \cdot 3$ & $36117 \cdot 9$ \\
$\mathrm{HD}$ & $36406 \cdot 2$ & $36405 \cdot 5$ \\
$\mathrm{D}_{2}$ & 36748.9 & $36748 \cdot 2$ \\
\hline
\end{tabular}


ment? My own answer to this is: at present, seldom. I am now speaking of properties depending on the electronic motion. There is an important point to make here. A number, whether coming from theory or experiment, is really useless if we have no idea of its accuracy. We have at present no practical, reliable method for calculating error bounds for quantum mechanical quantities (except upper bounds for total energy). The only validation for a given approximate method is the agreement with experiment. This places greater emphasis on the one hand upon the availability of reliable and accurate experimental data and on the other hand on the application of the method to a large and varied group of substances. Too often, in my opinion, a method is tried out on a very small group of molecules and extended claims are based on the agreement with experiment, which can very well be fortuitous.

Another obstacle to the utilization of quantum mechanical methods is that, for many quantities, purely empirical rules give more accurate results. For example, it is in general better to transfer bond distances from one molecule to a similar one than to calculate them $a$ priori. It is also vastly simpler. Similarly, additivity rules for bond energies, bond dipole moments, atomic polarizabilities, etc. usually give better results than available a priori methods for polyatomic molecules. See Table 3.,8

Naturally, there are problems which are inaccessible or partly inaccessible to experiment, such as, for example, the determination of potential energy surfaces for chemical reactions. For these, theoretical calculations may be the only available approach despite their uncertainty.

Some harm has probably been done to the field of quantum chemistry by exaggerated claims regarding its present capability for replacing experiment. The subject has plenty of importance without this kind of exaggeration.

Has quantum mechanics been important to chemistry? Despite my somewhat negative views on the present

Table 3. Some properties calculated via a priori MO theory

\begin{tabular}{|c|c|c|c|}
\hline \multirow[b]{2}{*}{ Molecule } & \multicolumn{2}{|c|}{ Geometries (STO-3G) ${ }^{7}$} & \multirow[b]{2}{*}{ Obs. } \\
\hline & Parameter & Calc. & \\
\hline \multirow[t]{2}{*}{$\mathrm{C}_{6} \mathrm{H}_{6}$} & $r_{\mathrm{CH}}$ & $1 \cdot 08$ & $1 \cdot 084$ \\
\hline & $r_{\mathrm{CC}}$ & $1 \cdot 39$ & $1 \cdot 397$ \\
\hline \multirow[t]{2}{*}{$\mathrm{CO}_{2}$} & $r_{\mathrm{CO}}$ & $1 \cdot 188$ & $1 \cdot 160$ \\
\hline & $\theta_{\text {oco }}$ & $0 \cdot 0^{\circ}$ & $0 \cdot 0^{\circ}$ \\
\hline \multirow[t]{3}{*}{$\mathrm{F}_{2} \mathrm{~N}_{2}$} & $r_{\mathrm{NF}}$ & $1 \cdot 277$ & $1 \cdot 384$ \\
\hline & $r_{\mathrm{NN}}$ & $1 \cdot 373$ & $1 \cdot 214$ \\
\hline & $\theta_{\mathrm{FNN}}$ & $111 \cdot 5^{\circ}$ & $114 \cdot 5^{\circ}$ \\
\hline $\mathrm{CF}_{4}$ & $r_{\text {CF }}$ & $1 \cdot 366$ & $1 \cdot 317$ \\
\hline \multirow[t]{2}{*}{$\mathrm{O}_{3}$} & $r_{\text {Oo }}$ & $1 \cdot 285$ & $1 \cdot 277$ \\
\hline & $\theta_{\text {ooo }}$ & $116 \cdot 2^{\circ}$ & $116 \cdot 8^{\circ}$ \\
\hline \multicolumn{4}{|c|}{ Dipole moments (extended basis) $^{8}$} \\
\hline $\mathrm{H}_{2} \mathrm{O}$ & & $2 \cdot 522$ & 1.846 \\
\hline $\mathrm{NH}_{3}$ & & $2 \cdot 105$ & $1 \cdot 468$ \\
\hline $\mathrm{CH}_{3} \mathrm{~F}$ & & $2 \cdot 408$ & $1 \cdot 855$ \\
\hline $\mathrm{H}_{2} \mathrm{CO}$ & & $3 \cdot 073$ & $2 \cdot 339$ \\
\hline $\mathrm{HCN}$ & & $3 \cdot 244$ & $2 \cdot 986$ \\
\hline \multicolumn{4}{|c|}{ Stretching force constants $(F x y)(\mathrm{STO}-3 \mathrm{G})^{7}$} \\
\hline $\mathrm{CH}_{4}$ & & $7 \cdot 4$ & $5 \cdot 8,5 \cdot 2$ \\
\hline $\mathrm{CH}_{3} \mathrm{~F}$ & & $9 \cdot 1$ & $5 \cdot 8,5 \cdot 7$ \\
\hline $\mathrm{CO}_{2}$ & & $26 \cdot 9$ & $18 \cdot 8$ \\
\hline $\mathrm{CF}_{4}$ & & $10 \cdot 4$ & $9 \cdot 2$ \\
\hline
\end{tabular}

capability for replacing experiment, my answer to this question is a resounding yes.

First, some of our most important instruments would probably not have been invented when they were if quantum mechanics had not been available. I would nominate nuclear magnetic resonance, the laser and therefore laser Raman, and certainly microwave spectroscopy as examples. Further, we could extract relatively little information from microwave spectroscopy without this theory, and that holds also for electron spectroscopy.

Secondly, the chemist's vocabulary has been strongly influenced. Just in rationalizing and discussing his results, words such as orbitals, resonance, hybridization, tunnelling, etc. are built into his language.

The most important contributions no doubt have come from the qualitative ideas. One set of these is contained in Pauling's classic book, The Nature of the Chemical Bond, ${ }^{9}$ originally published in 1938. This work has had an immense impact on chemistry. It stressed the so-called valence bond approach and the concepts of resonance, partial ionic character, overlap of atomic orbitals, hybridization and the directional properties of orbitals and covalent bonds, electronegativity of atoms, constancy of empirical bond lengths and bond energies, the hydrogen bond, and a remarkable set of principles for the structure of complex ionic crystals. The main theme was the concept of resonance.

It is an interesting paradox that in the subject index of The Nature of the Chemical Bond under orbital one does not find listed the term molecular orbital, although the concept is mentioned on one page. Yet surely this is the most basic contribution of quantum mechanics to chemistry. It is a remarkable aspect of the history of chemistry that these two approaches-the valence bond and the molecular orbital approximations-could both have had so much impact and yet seem so different. Even today chemists tend to mix the two in their discussions. It is only quite recently that their close relationship has been made fully clear.

Just as the the names of Slater, Pauling, van Vleck, Heitler, London, and Eyring loom large in the development of the valence bond approach, so do those of Hund, Mulliken, Hückel, Coulson, and Lennard-Jones appear on any list of contributors to molecular orbital theory. Some contributed to both.

Another qualitative idea of considerable significance is that of the correlation diagram. Mulliken made very important use of this in connection with the electronic states of diatomic molecules. ${ }^{10}$ His diagrams showed how the molecular orbitals of the separated atoms connected with those of the united atoms with the situation for the molecule being in between. This is an excellent way of codifying and rationalizing a considerable amount of experimental data. See Fig. 1. ${ }^{11}$

Walsh, in an influential series of papers in 1953, introduced correlation diagrams showing the expected variation in the energies of molecular orbitals with angles in, for example, $\mathrm{AH}_{2}, \mathrm{AB}_{2}, \mathrm{AB}_{3}$, etc. molecules. ${ }^{12}$ See Fig. 2. ${ }^{12}$ These led to rules concerning the qualitative geometry of simple molecules, involving only the number of valence shell electrons. These have been remarkably useful.

More recently the orbital correlation diagrams of Woodward and Hoffmann ${ }^{13}$ have had an immense impact. These show the connection of the molecular orbitals of the reactant with those of the product for concerted reactions of symmetric molecules. 


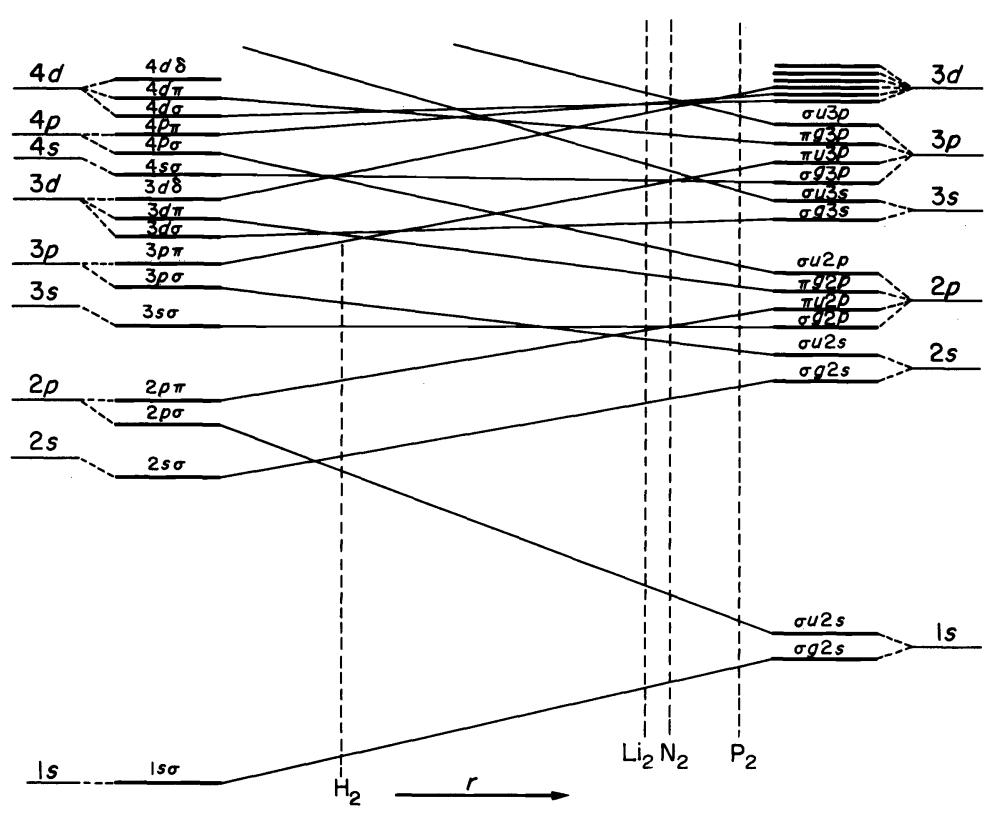

Fig. 1. Correlation diagram for homonuclear diatonic molecules. Orbital energies are schematically plotted against internuclear separation, from the "united atom" to separated atoms."

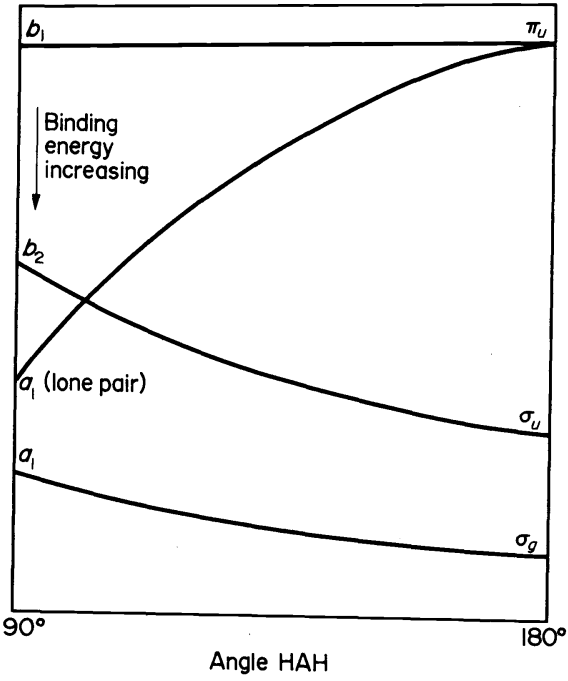

Fig. 2. Walsh diagram for HAH molecules. Orbital energy is plotted against bond angle. ${ }^{12}$

In qualitative molecular orbital theory the notions of bonding, non-bonding, and antibonding molecular orbitals should be mentioned; also the idea of localized molecular orbitals.

Ligand field theory is central in the present day discussions of transition metal chemistry. This technique clearly stems from the extensive development of crystal field theory by van Vleck, ${ }^{14}$ especially with respect to magnetic properties. His work in turn utilized the pioneer paper by Bethe ${ }^{15}$ on the application of group theory to the splitting of energy terms by crystal fields of various symmetries. This line of activity is a very elegant example of the importance of symmetry in quantum chemistry. Permutation symmetry enters through the Pauli Principle (and this use has been revived via "spin free" treatments).
Then geometric symmetry was applied to crystal term splitting, molecular vibrations, valence bond and molecular orbital treatments, rotational energy levels, nuclear spin statistical weights, molecules with internal rotations and inversions, reactions of symmetrical molecules and so on up to and including the present.

The highly quantum mechanical concept of tunnelling is beginning to have more applications to chemistry and is of special interest because it is a phenomenon with no classical analog. Tunnelling is involved in the well known inversion spectrum of ammonia- which led to the discovery of the ammonia maser and from that in several stages to the laser, and may lead to fusion power. Methyl groups undergo internal rotation by tunnelling from one energy minimum to another and this is the basis for the main method of determining barriers to internal rotation. Generally, one is interested in tunnelling either at low temperatures where processes going up and over a potential barrier are inhibited or in spectroscopy where one can examine low energy levels separately. Double minimum hydrogen bonds can exhibit tunnelling and this may have some importance. The tunnelling of hydrogen atoms through barriers in reaction mechanisms has generated controversy for decades.

Not all the chemical impact has come from qualitative applications. Numerical calculations ranging in rigor from Hückel computations to computer exploits involving the interaction of thousands of configurations have been important, too. At the lower levels, the quantitative results are often not as accurate as can be obtained from various simple empirical rules, but nevertheless these calculations have provided an essential guidance for the qualitative concepts. From them we know what the molecular orbitals look like for a lot of molecules. See Fig. $3 .^{16}$ Further, the search for practical approximations of reasonable accuracy cannot be successful if continuing efforts are not made to explore all kinds of approaches numerically. In the last five years real progress have been made with a priori SCF calculations with limited basis 


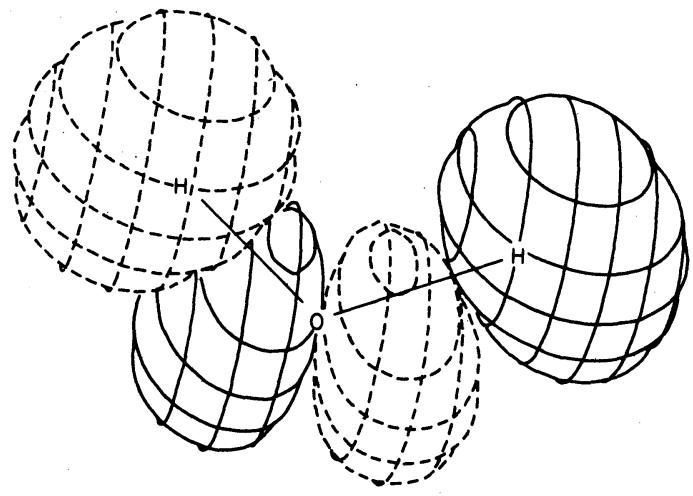

Fig. 3. Three-dimensional map for $2 \mathrm{~B}_{2}$ molecular orbital of water molecule. Shown are contour surfaces at one absolute value of orbital. Dashed lines are for negative value of MO. ${ }^{16}$

sets. For some properties these give quite respectable results. See Table 3.

The very elaborate configuration interaction calculations at a high level of accuracy are still restricted to quite small molecules but there is progress there in learning to choose appropriate basis sets and configurations and in improvements in computer utilization. In addition, computers continually become more powerful.

Although ideally one may yearn for studies in which clear cut predictions are made on large numbers of molecules of a variety of types and these independent predictions are then tested against later experimental results, we usually must settle for a good deal less. In many calculations considerable parameter juggling has occurred, and suspicions may well arise that certain molecules were not included in the list because they didn't come out well. Nevertheless, there is a psychological need for a theoretical framework on which to hang experimental data and to serve as a basis for planning new experiments. Often no great demands are put on a theory to make reliable predictions. So in this sense even a very bad theory can be better than no theory at all.

Can many-particle wavefunctions be replaced by simpler quantities? In many-electron systems, correlated wavefunctions such as those obtained by configuration interaction calculations are complicated and difficult to visualize, tabulate, or interpret. Much, but not all, of the information they contain can be condensed to much simpler form in terms of the electron density function $\rho(x, y, z)$. For the simple molecule water there are ten electrons and the wave function is then a function of thirty coordinates, not to mention the ten spins. This is a mathematical nightmare.

The density, on the other hand, is a function of ordinary three dimensional space and gives the number of electrons per unit volume for the various values of $x y z$. It can readily be calculated from $\psi$, but not vice versa. Figure 4 shows the density for water, for positions in the plane containing the three nuclei. ${ }^{17}$

The density can be used to calculate many, but not all, molecular properties. The suitable properties are those whose operators are purely multiplicative and spin free, such as the dipole moment, gradient at a nucleus, etc. A very important one is the force on a nucleus from the electronic charge. The force is equal to $-\partial E / \partial x$ where $E$ is the electronic energy and $x$ a cartesian coordinate of a nucleus. The famous Hellmann-Feynman theorem ${ }^{18,19}$ proves that this quantum-mechanical property is just equal to the classical electrostatic force on a nucleus due to the given charge distribution $\rho(x, y, z)$.

If we are given $\rho$ as a function of the nuclear coordinates, the Hellmann-Feynman force can be integrated to get the electronic energy change for a finite displacement of the nuclei. Thus, in principle, the electron density could be used to obtain dissociation energies, activation energies, barriers to internal rotation, vibration frequencies, etc. Since in chemistry, it is really only changes in energy that are important, this property of $\rho$ could be of importance.

However, for accurate energy changes, $\rho$ must be quite accurate. Whereas it is hard to see differences in the plots of $\rho$, such as Fig. 4 ; based on elaborate $\psi$ 's or very crude $\psi$ 's, the forces computed from the densities from approximate wavefunctions can be very bad.

There is another connection between the density and the energy. Long ago $^{20}$ the Thomas-Fermi statistical atom model used an approximation in which the total energy is taken to be the integral over the three-dimensional space of contributions from each local volume element. Then each local volume element is assumed to contribute the same energy as it would if it were part of an infinite uniform electron gas with the given local density $\rho(x y z)$. Since the energy of a uniform gas as a function of its density is pretty well known, this gives an approximate way of calculating the energy of a non-uniform electron distribution. Hohenberg and $\mathrm{Kohn}^{21}$ showed that there must exist a relation between the electron density function and the energy but they did not say what the relation is.

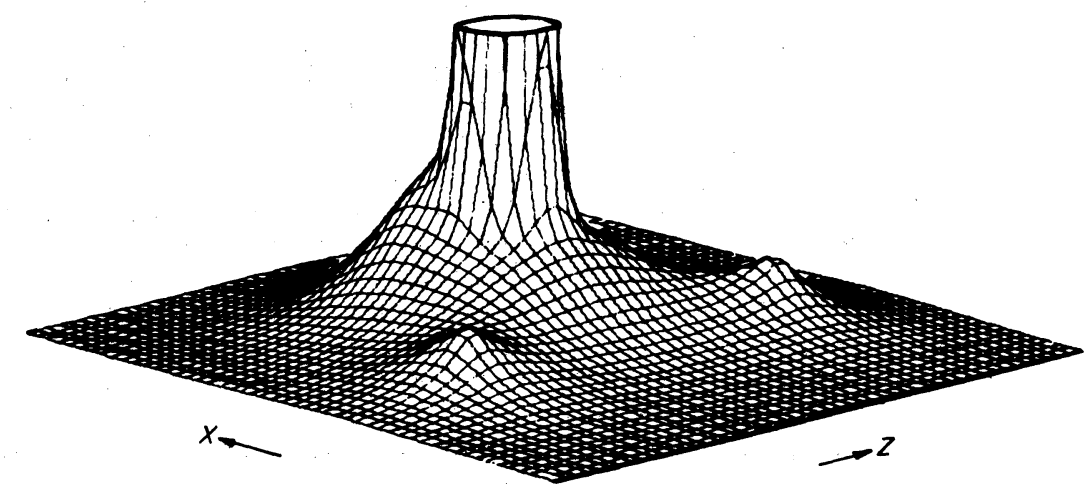

Fig. 4. Electron density plot for $\mathrm{H}_{2} \mathrm{O}$, in the plane of the nuclei. Peak at oxygen has been truncated. ${ }^{17}$ 
The "local energy" approximation outlined above is not really an accurate one but Gordon and $\mathrm{Kim}^{22}$ and others have recently found good uses for it nonetheless, for example in calculating repulsive forces between rare gases.

The main difficulty with the electron density approach is that we don't know how to obtain $\rho$ except from wavefunctions. If we knew accurately how the energy depended upon the density, the variational principle could be used, but this does not work well with the local energy approximation.

The density cannot be employed directly to calculate expectation values which involve non-multiplicative operators, for example the kinetic energy, but there is a related quantity which can be used. This is the onè-particle reduced density matrix,,$^{23} \Gamma^{(1)}\left(1,1^{\prime}\right)$, which is a function of two sets of coordinates and spins, 1 and $1^{\prime}$. This is still a great deal simpler than a many particle wavefunction. If we set $1^{\prime}$ equal to 1 and sum over the spins, we obtain $\rho$, the density, i.e.

$$
\rho(x y z)=\sum_{s=-1 / 2}^{1 / 2} \Gamma^{(1)}(x, y, z, s, x, y, z, s) .
$$

This function $\Gamma^{(1)}$ can be expanded in products of pairs of spin-orbitals. If the orbitals are properly chosen, it can be expressed in the form

$$
\Gamma^{(1)}\left(1,1^{\prime}\right)=\sum_{k} \lambda_{k} \chi_{k}(1) \chi_{k}^{*}\left(1^{\prime}\right)
$$

where the $\chi_{k}$ are a certain set of spin orbitals called natural spin-orbitals while the coefficients $\chi_{k}$ are called occupation numbers. Here then is a way of condensing and codifying at least much of the information in our most elaborate wavefunctions in a manner closely analogous to the simplest orbital descriptions. For ground state closed shell molecules (with $N$ electrons) near their equilibrium configurations, it seems to be true so far that $N / 2$ natural orbitals have occupation numbers near unity. I have called these the major natural orbitals. The rest, the minor natural orbitals, seem to have quite small occupation numbers, say 0.01 or less. They must not be neglected, however, for correlation effects.

For a single determinant wavefunction such as a restricted Hartree-Fock $\psi$ there are just $N / 2$ natural orbitals all together and each has occupation number unity. The natural orbitals are then, for this $\psi$, the Hartree-Fock orbitals or any unitary transformation of them.

I do not intend to pursue this topic further here but will only say that I have been trying to develop a paraphrase of ordinary qualitative molecular orbital theory which talks about natural orbitals instead of ordinary MO's and about occupation numbers instead of orbital energies in correlation diagrams. The purpose is to build a picture of molecules which is related to much more accurate wavefunctions than is conventional MO theory, and yet is as close as possible to the standard picture, which has proved to be so useful.

The two-particle reduced density matrix $\Gamma^{(2)}\left(1,2 ; 1^{\prime}, 2^{\prime}\right)$ can also be obtained from the wavefunction ${ }^{23}$ and contains more of the information from $\psi$ than does $\Gamma^{(1)}$. It can be used, for example, to calculate the expectation values of all one and two particle operators. This includes $H$, the energy, so in principle one has hoped to apply the variational principle directly and thus bypass the wavefunction altogether, finding instead this density matrix so as to minimize the energy. Unfortunately this appears to be an illusion since the auxilliary conditions, the so-called $N$-representability conditions, required by the Pauli Principle, are so numerous and complicated. ${ }^{24,25}$ I fear this approach is impractical. However, the two-particle density matrix can be put in a standard diagonal form (in terms of two particle natural spin geminals) and this is useful for comparing different approximate wavefunctions for the same problem.

Are Hartree-Fock wavefunctions good enough? It is well known that a single determinant wavefunction, i.e. the restricted Hartree-Fock $\psi$, yields an enormous absolute error in the total energy, even though the percentage error can be fairly small. For example, in water the correlation energy or the difference between Hartree-Fock and the true energy corrected for relativity is about $230 \mathrm{kcal}^{26}$ or $\sim 0.48$ of $1 \%$, whereas even one or two kilocalories can be of interest to chemists. Despite this, Hartree-Fock wavefunctions give reasonably good predictions for many properties, even certain energy differences. Although there are some considerations suggesting that one-electron properties might be expected to come out fairly well, it has to be admitted that the reasonably successful performance of near Hartree-Fock $\psi$ 's is an empirical observation. This means that we need many more calculations and comparisons with experiment before we can be confident that we know the range of applicability of this approximation. This, in my opinion, is a very important task that needs much further work.

We do know that correlation energy is important for really accurate predictions, especially for energies. Even qualitatively, Hartree-Fock fails in certain circumstances. A well known case is the incorrect dissociation of molecules which yield open shell atoms. Examples include $\mathrm{H}_{2}$ and $\mathrm{F}_{2}$. In the case of $\mathrm{F}_{2}$, Hartree-Fock gives no binding at all.

To correct this particular limitation qualitatively requires the addition of terms representing the correct atomic dissociation products, in other words a very limited configuration interaction, but for accurate energies very large numbers of configurations may be required.

Another situation where the mixing of configurations can be expected to be important is in the description of many excited states because the density of states can be greater at higher energies.

A special case where configuration mixing is important is in the description of the transition complex for a "forbidden" concerted reaction, where the system is changing from one set of orbitals to another.

What has happened to the valence bond method? There is no doubt that almost all current quantum mechanical calculations of molecules are based on molecular orbitals. Does this mean that the valence bond method is dead? Certainly it is not in the sense that chemists still use Lewis dot structures and still talk about resonance. However, numerical calculations have been rare. In recent years, though, the valence bond method has been somewhat revived, particularly by the work of Goddard ${ }^{27}$ In his calculations, he replaces the rigid atomic orbitals of the original valence bond wavefunctions by variationally determined orbitals. If we write a single determinant restricted Hartree-Fock $\psi$ in the form

$$
\psi=A a(1) a(2) b(3) b(4) \ldots \alpha(1) \beta(2) \alpha(3) \beta(4) \ldots
$$

where $A$ is the antisymmetrizer, $a, b, \ldots$ are molecular 
orbitals doubly occupied and $\alpha, \beta$, are the spins, then we know that $\psi$ is unchanged if we replace $a, b$, etc. by any linear combination of $a, b, \ldots$ In particular the new linear combination may be chosen to localize the molecular orbitals in some sense.

Next we may replace some or all of the pairs $a(1) a(2)$, etc. by Heitler-London pairs such as

$$
a(1) a(2) \rightarrow A(1) B(2)+B(1) A(2)
$$

(this is singlet pairing). If the new "atomic" orbitals $A$ and $B$ are variationally determined, the resulting $\psi$ cannot be worse than Hartree-Fock because the case $A=B=a$ is included among the possibilities variationally tested and this choice reduces back to Hartree-Fock. In general, such "splitting" of the Hartree-Fock orbitals will give lower energy, i.e. some of the correlation energy. Further, if the pairs involving the valence bonds are split, the wavefunction will now behave correctly as the molecule dissociates.

Obviously in this sense the so-called generalized valence bond (GVB) method is not too far from Hartree-Fock. Further by writing

$$
\begin{aligned}
A(1) B(2)+B(1) A(2)= & C_{1}(A(1)+B(1))(A(2)+B(2)) \\
& +C_{2}(A(1)-B(1))(A(2)-B(2))
\end{aligned}
$$

in which $C_{1}$ and $C_{2}$ depend on the overlap of $A$ and $B$, we see that the Heitler-London pair can be expressed in terms of two localized MO configurations, one with a bonding orbital $(A+B)$, the other with an antibonding MO $(A-B)$.

If $A$ and $B$, while not orthogonal to each other, are further restricted to be orthogonal to all the other orbitals in $\psi$, a special form of the strongly-orthogonal pair wavefunction is obtained. Apparently, even if this orthogonality condition is not artifically imposed, the variation process causes the orbitals to tend toward this orthogonality, at least approximately.

It also seems that the variational flexibility provided to the orbitals in the GVB method takes care automatically of much of the resonance energy. Resonance structures normally involve different ways of pairing the same set of orbitals, i.e. different spin assignments to the orbitals. The GVB method can be extended to include all such pairing with variationally determined coefficients but adding this feature does not appear to lower the energy very much, suggesting that the variation of the orbitals takes care of most of the resonance stabilization, in many cases.

So the valence bond is not dead but it does involve more work because it has one orbital per electron instead of one for each pair of electrons. Goddard ${ }^{27}$ is attempting to develop it for qualitative uses, just as MO theory is so often used.

What about scattering? In the early days, quantum chemistry was largely confined to stationary states, but that is clearly no longer the case. In particular there has recently been an immense interest in scattering problems. This has gone hand in hand with the experimental development of molecular beam techniques. The scattered particles may be photons, electrons, neutrons, atoms or molecules and scattering may be elastic or inelastic and may or may not involve chemical reaction. The theory used may be classical, semi-classical or completely quantum-mechanical. Certainly this is a tremendously active area of current research and one which I cannot treat properly in this short review.
What about the future? I claim no powers as a soothsayer. It does seem reasonable to expect more progress in computers, both in speed and cost. I don't think the miracle of the integrated circuit is the last miracle from solid state physics. But the difficulty rises so rapidly with number of electrons that I doubt if increases in computer capability alone will extend accurate calculations to very much larger molecules. Of course, someone may ultimately come up with an effective really new approach, but the history of quantum chemistry does not make for optimism on this score. Hartree developed his method even before quantum mechanics, and it is still basic. Configuration interaction is also very old.

What is new is the computer and increasing skill in using it. The details about basis functions, choice of orbital exponents, selection of configurations, methods of calculating integrals, etc. are what make all the difference. Sometimes I think there probably are good methods which have been discarded because they were never tested by someone really skilled with computation.

One can well speculate about the possibility that the advent of the computer age led to the partial substitution of computing for thinking. There have been indeed relatively few really new ideas in the last twenty years. This may mean that there is nothing there to discover, or that the really original minds are occupied in other areas, or that the computer, for all its wonderful accomplishments, has diverted people. Perhaps the truth is some linear combination of all of these.

I certainly hope there will be radically new and better schemes. In particular it has never seemed right to me that one has to add and subtract thousands of large numbers to get small differences. One development which is greatly needed is a practical method for calculating error bounds. Even a very approximate scheme would be most useful.

Perhaps an accurate functional giving the energy in terms of the electron density will be found so that densities will be obtained directly, thus bypassing wavefunctions for many purposes. That really could be revolutionary.

Finally, it is likely that qualitative considerations will continue to dominate the applications of quantum chemistry. Chemistry has a method of making progress which is uniquely its own and which is not understood or appreciated by non-chemists. Our concepts are often ill-defined, our rules and principles full of exceptions, and our reasoning frequently perilously near being circular. Nevertheless, combining every theoretical argument available, however shaky, with experiments of many kinds, chemists have built up one of the great intellectual domains of mankind and have acquired great power over nature, for good and for ill. My guess is that they will continue to work in this way and that any new results and methods from quantum theory will be built into the structure just as the old ones, errors and all, have been. One thing is sure-the rate of progreess in quantum chemistry today is very high and whatever its capabilities are today, they will be notably more impressive in the near future.

\section{REFERENCES}

${ }^{1}$ P. A. M. Dirac, Proc. Roy. Soc. A123, 714 (1929).

${ }^{2}$ A. Veillard and E. Clementi, J. Chem. Phys. 49, 2415 (1968)

${ }^{3}$ G. M. Schwenzer, D. H. Liskow, H. F. Schaefer, III., P. S

Bagus, B. Liu, A. D. McLean and M. Yoshimine, J. Chem. Phys. 58, 3181 (1973).

${ }^{4}$ H. M. James and A. S. Coolidge, J. Chem. Phys. 1, 825 (1933)

${ }^{5}$ W. Kolos and L. Wolniewitz, J. Chem. Phys. 41, 3663 (1964); 49 404 (1968). 
${ }^{6}$ G. Herzberg, J. Mol. Spectro. 33, 147 (1970).

${ }^{7}$ M. D. Newton, W. A. Lathan, W. J. Hehre and J. A. Pople, J. Chem. Phys. 52, 4064 (1970).

${ }^{8}$ R. Ditchfield, W. J. Hehre and J. A. Pople, J. Chem. Phys. 54, 724 (1971).

${ }^{9}$ Linus Pauling, The Nature of the Chemical Bond. Cornell Univ. Press, Ithaca (1939).

${ }^{10}$ R. S. Mulliken, Rev. Mod. Phys. 4, 1 (1932).

${ }^{11}$ G. Herzberg, Molecular Spectra and Molecular Structure I. 2nd ed., p. 328. Van Nostrand Reinhold, New York (1950).

${ }^{12}$ A. D. Walsh, J. Chem. Soc. 2260 (1953).

${ }^{13}$ R. Hoffmann and R. B. Woodward, J. Amer. Chem. Soc. 87, 2046, 4388, 4389 (1965).

${ }^{14} \mathrm{~J}$. H. van Vleck, The Theory of Electric and Magnetic Susceptibilities. Sec. 73. Oxford Press (1932).

${ }^{15}$ H. Bethe, Ann. Phys. 5, 133 (1929).
${ }^{16} \mathrm{~W}$. L. Jorgensen and L. Salem, The Organic Chemist's Book of Orbitals. p. 70. Academic Press, New York (1973).

${ }^{17} \mathrm{C}$. W. Kern and M. Karplus, in Water-A Comprehensive Treatise. Vol. 1, Chap. 2, (ed. F. Franks) Plenum, New York. ${ }^{18} \mathrm{~J}$. Hellmann, Einführung in die Quantenchemie. Deuticke, Leipzig (1937).

${ }^{19}$ R. Feynman, Phys. Rev. 56, 340 (1939).

${ }^{20}$ N. H. March, Adv. Phys. 6, 1 (1957).

${ }^{21}$ P. Hohenberg and W. Kohn, Phys. Rev. 136, B864 (1964).

${ }^{22}$ R. G. Gordon and Y. S. Kim, J. Chem. Phys. 56, 3122 (1973). ${ }^{23}$ P. O. Löwdin, Phys. Rev. 97, 1474 (1954).

${ }^{24}$ F. Weinhold and E. B. Wilson, J. Chem. Phys. 47, 2298 (1967).

${ }^{25}$ E. R. Davidson, J. Math. Phys. 10, 725 (1969).

${ }^{26} \mathrm{~W}$. Meyer, Int. J. Quantum Chem. 55, 341 (1971).

${ }^{27}$ W. A. Goddard, III, T. H. Dunning, Jr., W. J. Hunt and P. J. Hay, Accts. Chem. Res. 6, 368 (1973). 